\title{
Protein Synthesis on Demand, and Make it Snappy!
}

Category

Flow Chemistry

Key words

automated synthesis

protein synthesis

barstar

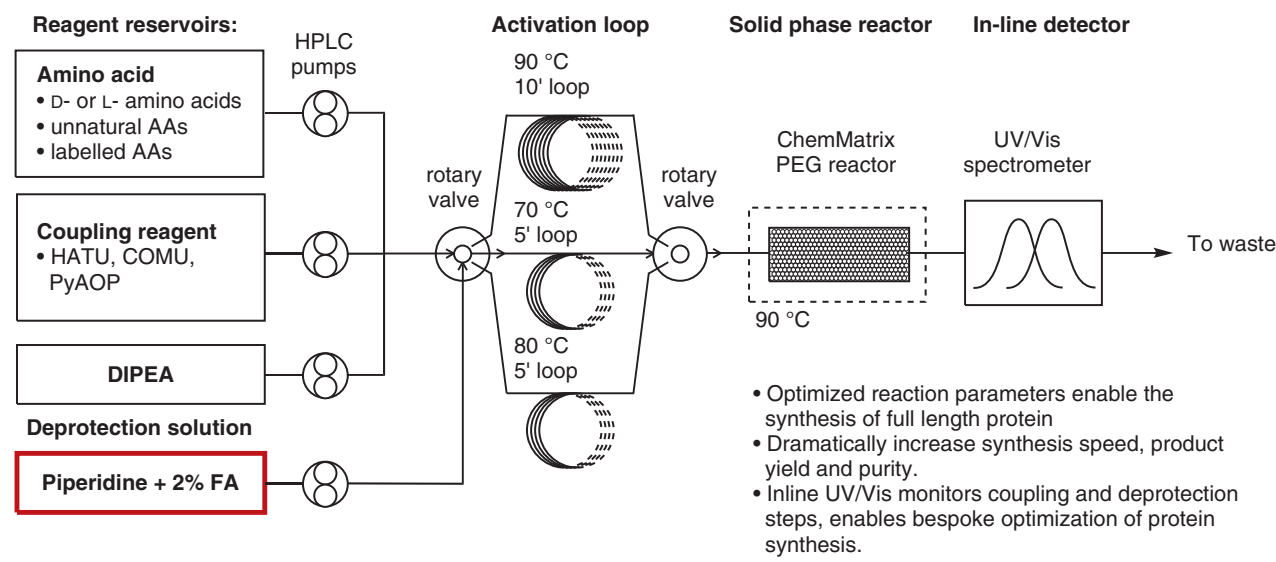

Representative proteins synthesized by automated fast-flow peptide synthesis (AFPS):

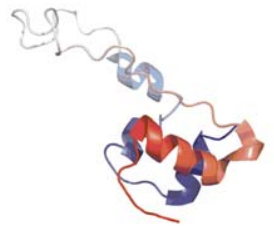

Sequence length: Synthesis time:

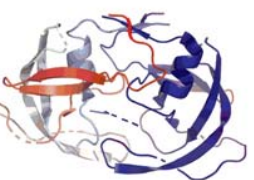

HIV protease 99 AA $4.5 \mathrm{~h}$ (5.3 mg, $1 \%$ yield)

$$
\begin{gathered}
\text { vs. } \\
47 \mathrm{~h}
\end{gathered}
$$

(complex mixture) using standard SPPS
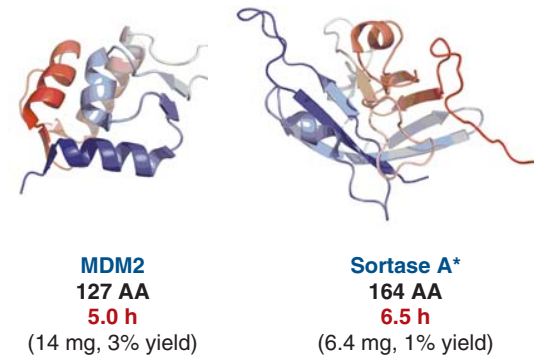

Sortase A
164 AA

$6.4 \mathrm{mg}, 1 \%$ yield
Significance: Solid-phase peptide synthesis (SPPS) is routinely performed for the preparation of small peptides. Despite decades of optimization, synthesis of peptides with sequences longer than 50 amino acids is challenging due to the generation of byproducts stemming from deletion, epimerization, and truncation. The chemical synthesis of fulllength proteins did not become a practical reality until the introduction of native chemical ligation $(\mathrm{NCL})$, which often requires conditions specific to the protein. Pentelute and co-workers report an optimized protocol using their automated fast-flow peptide synthesis (AFPS) system that is capable of synthesizing full-length proteins of up to $164 \mathrm{ami}$ no acids, without the use of chemical ligation techniques, on time-scales that rival recombinant expression.
Comment: To synthesize full-length proteins, each step in the peptide coupling reaction had to be carefully optimized. The critical variables the authors identified included preactivation temperature and time, reaction temperature and Fmoc-deprotection conditions. The reaction coupling efficiency could be indirectly evaluated using an in-line UV/ Vis spectrometer. AFPS was capable of synthesizing full-length proteins 10 times faster than traditional SPPS, with higher overall yield and purity. After purification and folding, the synthesized proteins possessed the same biological activity and biophysical characteristics as recombinant proteins. By using this technology, unnatural AAs can be easily incorporated and site-specific mutations are possible. 